\title{
Value of contrast-enhanced CT based radiomic machine learning algorithm in differentiating gastrointestinal stromal tumors with KIT exon 11 mutation: a two-center study
}

Bo Liu

Department of General Surgery; The Affiliated Hospital of Qingdao University

Hexiang Wang

Department of Radiology; The Affiliated Hospital of Qingdao University

Shunli Liu

Department of Radiology; The Affiliated Hospital of Qingdao University

Shifeng Yang

Shandong Provincial Hospital Affiliated to Shandong First Medical University

\section{Yancheng Song}

Department of General Surgery; The Affiliated Hospital of Qingdao University

Hao Liu

Department of General Surgery; The Affiliated Hospital of Qingdao University

\section{Ziwen Zheng}

Department of General Surgery; The Affiliated Hospital of Qingdao University

Junjiang Zhao

Department of General Surgery; The Affiliated Hospital of Qingdao University

\section{Feng Hou}

Department of Pathology; The Affiliated Hospital of Qingdao University

\section{Xuelong Jiao}

Department of General Surgery; The Affiliated Hospital of Qingdao University

Jian Zhang ( $\nabla$ zhangjian@qduhospital.cn )

Department of General Surgery; The Affiliated Hospital of Qingdao University

https://orcid.org/0000-00032998-873X

\section{Research}

Keywords: Gastrointestinal stromal tumor, Contrast-enhanced CT, Machine learning radiomics, KIT mutation, Mutation analysis

Posted Date: August 4th, 2020

DOl: https://doi.org/10.21203/rs.3.rs-48361/v1 
License: (c) (1) This work is licensed under a Creative Commons Attribution 4.0 International License. Read Full License

Version of Record: A version of this preprint was published at Diagnostic and Interventional Radiology on December 30th, 2021. See the published version at https://doi.org/10.5152/dir.2021.21600. 


\section{Abstract}

Background Knowing the genetic phenotype of gastrointestinal stromal tumors (GISTs) is essential for patients who receive therapy with tyrosine kinase inhibitors.

Methods We enrolled 106 patients (80 in the training set, 26 in the validation set) with clinicopathologically confirmed GISTs from two centers. Preoperative and postoperative clinical characteristics were selected and analyzed to construct the clinical model. Arterial phase (A-phase), venous phase (V-phase), delayed phase (Dphase), and combined radiomics algorithms were generated from the training set based on contrastenhanced computed tomography (CE-CT) images. Various radiomics feature selection methods were used, namely least absolute shrinkage and selection operator (LASSO); minimum redundancy maximum relevance (mRMR); and generalized linear model (GLM) as a machine-learning classifier. Independent predictive factors were determined to construct preoperative and postoperative radiomics nomograms by multivariate logistic regression analysis. The performances of the clinical model, radiomics algorithm, and radiomics nomogram in distinguishing GISTs with the KIT exon 11 mutation were evaluated by area under the curve (AUC) of the receiver operating characteristic (ROC).

Results The combined radiomics algorithm was found to be the best prediction model for differentiating the expression status of the KIT exon 11 mutation ( $\mathrm{AUC}=0.836 ; 95 \%$ confidence interval $(\mathrm{Cl}), 0.640-0.951$ ) in the validation set. The clinical model, and preoperative and postoperative radiomics nomograms had AUCs of 0.606 ( $95 \% \mathrm{Cl}, 0.397-0.790), 0.715$ ( $95 \% \mathrm{Cl}, 0.506-0.873)$, and 0.679 (95\% Cl, 0.468-0.847), respectively, with the validation set.

Conclusion The radiomics algorithm could distinguish GISTs with the KIT exon 11 mutation based on CE-CT images and could potentially be used for selective genetic analysis to support the precision medicine of GISTs.

\section{Background}

Gastrointestinal stromal tumors (GISTs) are the most commonly diagnosed subepithelial tumors of the gastrointestinal tract (1-3). However, GISTs respond poorly to chemotherapy and prognosis prediction is challenging because of their biological diversity and histological heterogeneity (2). Mutations in the KIT gene, which encodes a tyrosine kinase, are known to lead to the development of GISTs. Besides KIT, mutations in other genes (e.g., platelet-derived growth factor receptor alpha (PDGFRA)) also have been associated with GISTs (4). The KIT mutations have been widely studied and targeted therapy with tyrosine kinase inhibitors (TKIs) has been developed and implemented successfully in the current treatment of GISTs. Different mutations in the different genes associated with GISTs have been shown to affect the response of GISTs to TKls $(2,5)$.

Most GISTs (75-85\%) have KIT mutations, 5-7\% have PDGFRA mutations, and $12-15 \%$ have no detectable mutation in KIT or PDGFRA $(1,4)$. The National Comprehensive Cancer Network (NCCN) guidelines highly recommend the genetic analysis of GISTs, because the expression status of specific regions of the KIT and PDGFRA gene sequences are correlated with a specific response to TKIs (2). The KIT exon 11 mutation, which 
accounts for about $75 \%$ of KIT mutations in GISTs, is the most common imatinib-target mutation and GISTs with this mutation respond well to standard imatinib therapy $(1,5-7)$. Because there are relatively few patients who do not have the KIT exon 11 mutation, genetic analysis is required to define a wide variety of treatment effects $(2,6,7)$. Therefore, before clinical treatment with TKIs, it is important to determine the expression status of the KIT exon 11 mutation. However, considering tolerance of the procedure and the heterogeneity of GISTs, conventional invasive biopsy or surgery to obtain specimens has many defects $(2,8$, 9). Further, because of the cost, routine genetic analysis of GISTs is still not performed widely $(10,11)$. Consequently, it is urgent to establish a non-invasive, repeatable, economical, and accurate pretreatment method to identify the expression status of the KIT exon 11 mutation.

Contrast-enhanced computed tomography (CE-CT) is a basic diagnostic method that is used to detect lesions and evaluate the treatment response of GISTs (10-12). Medical imaging has been playing an increasing key role in personalized precision medicine. Radiomics has been applied to oncology studies (13) and has been widely used in diagnosis (14), prognostic evaluation (15), prediction of biological behaviors (16), and even genetic prediction $(11,17)$.

Previous studies applied CT texture analysis with a small amount of single-center data to distinguish GISTs without the KIT exon 11 mutation (11). To our knowledge, the radiomics approach has not been evaluated for predicting GISTs with the KIT exon 11 mutation. Thus, the aim of this study was to develop and validate a triphase CE-CT based radiomics model with two-center data that can distinguish the patients with the KIT exon 11 mutation.

\section{Methods}

\section{Selection of Patients and Their Characteristics}

This retrospective study was approved by the Institutional Review Boards of the Affiliated Hospital of Qingdao University (institution 1) and the Shandong Provincial Hospital affiliated to Shandong First Medical University (institution 2), which waived patient informed consent. We screened the patients with pathologically confirmed GISTs in the databases of these institutions from July 2008 to August 2019. Patients who fulfilled the following inclusion criteria were included in the study: (a) definitive genetic analysis results could be obtained; (b) complete clinical characteristics could be obtained; (c) tri-phase CE-CT images before treatment (neoadjuvant therapy or surgery) could be obtained; and (d) the GIST size was $\geq 2 \mathrm{~cm}$ and could be outlined in the CE-CT images for volume of interest segmentation.

According to these criteria, 106 patients who also underwent genetic analysis between August 2010 and August 2019 were included: 93 were from institution 1 and 13 were from institution 2. Because the number of patients from institution 2 was small, their data could not be used as an external validation set alone. Therefore, the data of patients from both institutions were merged, then randomly allocated to training and validation sets in a 3:1 ratio. The number of patients with the KIT exon 11 mutation was 45, the number of patients without the KIT exon 11 mutation was 61 . Among the 106 patients, 57 were male and 49 were female (mean age $59.5 \pm 9.9$ years). Detailed information on the clinical characteristics of the selected patients is given in Table 1. A detailed description of the workflow process is shown in Fig. 1. 
Page 5/21 
Table 1

Clinicopathologic and Demographic Data

\begin{tabular}{|c|c|c|c|c|}
\hline Clinical factors & & $\begin{array}{l}\text { KIT exon } 11 \text { mutation } \\
(-), n=45\end{array}$ & $\begin{array}{l}\text { KIT exon } 11 \text { mutation } \\
(+), n=61\end{array}$ & $\begin{array}{l}\mathrm{P} \\
\text { value }\end{array}$ \\
\hline \multirow[t]{2}{*}{ Gender } & Male & 22 & 35 & \multirow[t]{2}{*}{0.386} \\
\hline & Female & 23 & 26 & \\
\hline $\begin{array}{l}\text { Age (Median[range]), } \\
\text { year }\end{array}$ & & $57(47.50-66.50)$ & $62(55.00-66.00)$ & 0.051 \\
\hline \multirow[t]{3}{*}{ Growth pattern } & Endoluminal & 12 & 20 & \multirow[t]{3}{*}{0.238} \\
\hline & Exophytic & 15 & 26 & \\
\hline & Mixed & 18 & 15 & \\
\hline $\begin{array}{l}\text { CEA(Median[range]), } \\
\mathrm{ng} / \mathrm{ml}\end{array}$ & & $1.76(1.14-2.91)$ & $2.00(1.18-3.16)$ & 0.623 \\
\hline $\begin{array}{l}\text { CA199(Median[range]), } \\
\text { ng/ml }\end{array}$ & & $10.61(6.22-17.07)$ & $8.64(5.66-13.82)$ & 0.215 \\
\hline $\begin{array}{l}\text { CA125(Median[range]), } \\
\mathrm{ng} / \mathrm{ml}\end{array}$ & & $10.06(7.56-27.23)$ & $9.06(7.55-11.52)$ & 0.144 \\
\hline $\begin{array}{l}\text { CA724(Median[range]), } \\
\mathrm{ng} / \mathrm{ml}\end{array}$ & & $4.40(1.24-17.59)$ & $1.49(1.07-3.91)$ & 0.006 \\
\hline $\begin{array}{l}\text { Maximum diameter of } \\
\text { tumor }\end{array}$ & & $5.00(3.50-8.00)$ & $5.00(3.85-8.85)$ & 0.468 \\
\hline \multirow{2}{*}{$\begin{array}{l}\text { Mitotic count } \\
\text { (mitoses/50 hpf) }\end{array}$} & $<5$ & 31 & 32 & \multirow[t]{2}{*}{0.08} \\
\hline & $\geq 5$ & 14 & 29 & \\
\hline \multirow[t]{4}{*}{ Anatomic location } & Stomach & 21 & 38 & \multirow[t]{4}{*}{0.109} \\
\hline & Small bowel & 18 & 20 & \\
\hline & Colorectum & 2 & 2 & \\
\hline & Mesentery & 4 & 1 & \\
\hline Ki-67(Median[range]), \% & & $5(3-11)$ & $10(5-20)$ & 0.070 \\
\hline \multirow[t]{2}{*}{ CD-117 } & $(-)$ & 5 & 0 & \multirow[t]{2}{*}{0.012} \\
\hline & $(+)$ & 40 & 61 & \\
\hline \multirow[t]{2}{*}{ CD-34 } & $(-)$ & 9 & 7 & \multirow[t]{2}{*}{0.226} \\
\hline & $(+)$ & 36 & 54 & \\
\hline DOG-1 & $(-)$ & 4 & 1 & 0.160 \\
\hline
\end{tabular}




\begin{tabular}{|c|c|c|c|c|}
\hline \multicolumn{2}{|l|}{ Clinical factors } & \multirow{2}{*}{$\begin{array}{l}\text { KIT exon } 11 \text { mutation } \\
(-), n=45 \\
41\end{array}$} & \multirow{2}{*}{$\begin{array}{l}\text { KIT exon } 11 \text { mutation } \\
(+), n=61\end{array}$} & \multirow[t]{2}{*}{$\begin{array}{l}P \\
\text { value }\end{array}$} \\
\hline & $(+)$ & & & \\
\hline \multirow{4}{*}{$\begin{array}{l}\text { NIH risk stratification } \\
(2008)\end{array}$} & Very low & 2 & 2 & \multirow[t]{4}{*}{0.444} \\
\hline & Low & 14 & 16 & \\
\hline & Intermediate & 10 & 13 & \\
\hline & High & 19 & 30 & \\
\hline
\end{tabular}

\section{Tri-phase CE-CT Image Screening and Volume of Interest Segmentation}

All 106 patient had undergone CE-CT scanning with 16 or 64-detector spiral CT scanners (Siemens Somatom Definition, GE Hispeed, GE Bright, or GE Optima 670). For all these measurements, the tube current was 200$240 \mathrm{mAs}$, the slice thickness was $5.0 \mathrm{~mm}$, the tube voltage was $120-160 \mathrm{kV}$, and the injection rate was $3.5 \mathrm{ml} / \mathrm{s}$, with intravenous injection of contrast (iopromide, $80 \mathrm{ml}$ ) and arterial, venous, and delayed phase with 30,70 , and 300 s delay, respectively.

ITK-SNAP (v3.6.0; www.itksnap.org) was used for three-dimensional volume of interest (VOI) segmentation of the CE-CT images. Radiologist 1, who had nine years' clinical experience of using ITK-SNAP, outlined the edge of the tumor on the tri-phase CE-CT images layer by layer and fused them into the VOI. The same radiologist repeated the VOI segmentation and feature extraction two weeks later. In addition, radiologist 2, who had 14 years' clinical experience of using ITK-SNAP, performed the VOI segmentation and feature extraction once. To evaluate inter- and intra-observer reproducibility, the two radiologists randomly selected 30 cases for VOI segmentation and feature extraction. Inter-/ intra-class correlation coefficients (ICCs) were used to evaluate the reproducibility and stability of radiomics feature extraction to identify robust radiomics features. The inter-observer ICC was derived from the first feature extraction by radiologist 1 and the feature extraction by radiologist 2. The intra-observer ICC was derived from the feature extraction performed twice by radiologist 1. Generally, ICCs $>0.75$ are considered to indicate good reproducibility or reliability. In this study, to ensure highly accurate features were selected, an ICC $>0.9$ was used as a criterion for good robustness.

\section{Image Normalization and Feature Extraction}

For the CE-CT regions, the $\mu \pm 3 \sigma$ method (18), gray-level quantization (19), and cubic interpolation (voxel size $=1 \times 1 \times 1 \mathrm{~mm} 3)(20)$ were used to redress the influences of distinct acquisition protocols, scales, and directions.

All the CE-CT images were processed using AnalysisKit software (v1.0.3; GE Healthcare, China) for texture feature extraction and quantification analysis of tissue heterogeneity. A total of 396 textural parameters were extracted, including the characteristics of 42 histograms, 10 Haralick features, 9 form factors, 48 grey-level 
co-occurrence matrix (GLCM) features with an offset of 1/4/7, and 60 gray-level run length matrix (GLRLM) features with an offset of $1 / 4 / 7$.

\section{Combat Compensation Method}

The distinguishing features of the texture patterns were retained using the combat compensation method (21), while eliminating the influence of the scanner and the protocol. This method was helpful for multi-center radiomics analysis (22) and can be used for CE-CT images.

\section{Clinical Characteristics}

The preoperative demographic and clinicopathologic data, including age, sex, and blood test results, were collected for the 106 patients. Postoperative data, including the location and size (maximum diameter) of the tumor, proteomics (e.g., expression statuses of tumor related proteins Ki-67, CD117, CD34, and D0G1), mitotic count, National Institutes of Health (NIH) risk stratification, growth pattern, and genetic phenotype, also were collected.

\section{Clinical Model, Radiomics Algorithm, and Radiomics Nomogram Building}

The relationship between clinical factors and the KIT exon 11 mutation was assessed by univariate logistic regression. Then, a multivariate logistic model was used with the significant clinical features $(P<0.05)$ to develop the clinical model.

The feature extraction algorithm implemented in the R statistical software (v3.6.3; www.Rproject.org) was used to select features and generate the models. To select the most valuable predictive factors in the training set, we incorporated the top 20 features of minimum redundancy maximum relevance (mRMR) into the least absolute shrinkage and selection operator method (LASSO) and fitted for regression of data. Generalized linear model (GLM), which is a machine-learning classifier, was trained with the training set using the 10-fold cross-validation approach. A radiomics algorithm was added with preoperative and postoperative clinical factors to build predictive nomograms for the training set with multivariable logistic regression analysis.

\section{Assessment and Validation of the Clinical Model, Radiomics Algorithm, and Radiomics Nomogram}

The effectiveness of the clinical model, radiomics algorithm, and radiomics nomogram in differentiating, calibration, and clinical value was verified with the validation set. The predictive capability was evaluated using the validation set by the receiver operating characteristic (ROC) curve, the area under the ROC curve (AUC), specificity, sensitivity, accuracy, and positive and negative predictive values. The calibration curves were used to assess the agreement between the predicted and actual results with the Hosmer-Lemeshow test (23). The clinical applicability of the radiomics algorithm and preoperative and postoperative radiomics nomograms also was assessed with decision curve analysis by calculating the net benefit at different threshold probabilities (24).

\section{Statistical Analysis}

R statistical software (v3.6.3; www.Rproject.org) was used for all the statistical analyses. 
To select the clinical factors, Fisher's exact test or the chi-square test was used to compare the categorical variables between the training and validation sets. The Mann-Whitney $\mathrm{U}$ test or independent t-test was used to evaluate the discrimination in clinical characteristics among different groups of continuous variables. The relationship between the KIT exon 11 mutation and clinical factors was assessed with univariate analysis.

\section{Results}

The 106 patients with GISTs were divided into two groups: those without the KIT exon 11 mutation and those with the KIT exon 11 mutation. The clinicopathologic and demographic data of these patients are summarized in Table 1. The expression status of CA724 and CD117 were significantly different between the two groups. No statistically significant differences were found between any of the other clinical characteristics.

The univariate logistic regression analysis of the clinical results showed that CA724 was significantly associated with GISTs with the KIT 11 mutation in the training set (Table 2). The multivariate logistic regression analysis of the clinical characteristics showed that CA724 $(P=0.004)$ and mitotic count $(P=$ 0.020) were independent predictors of the KIT exon 11 mutation in GISTs (Table 2). We constructed preoperative and postoperative clinical models on the basis of the multivariate logistic regression results. The performances of the preoperative (CA724) and postoperative (CA724 and mitotic count) clinical models in distinguishing GISTs with the KIT exon 11 mutation are shown in Table 4. For the preoperative clinical model with the training and validation sets, the AUCs were 0.669 and 0.509 respectively; for the postoperative clinical model with the training and validation sets, the AUCs were 0.770 and 0.606 respectively.

Table 2

Clinical Factors of Univariate \& Multivariate Logistic Regression Analysis for KIT Exon 11 mutation

\begin{tabular}{|lllllll|}
\hline & \multicolumn{2}{l}{ Univariate Analysis } & \multicolumn{4}{c|}{$\begin{array}{l}\text { Multivariate } \\
\text { Analysis }\end{array}$} \\
\hline $\begin{array}{l}\text { Clinical } \\
\text { Characteristics }\end{array}$ & OR & $95 \% \mathrm{Cl}$ & $\begin{array}{l}\text { P- } \\
\text { value }\end{array}$ & OR & $95 \% \mathrm{Cl}$ & $\begin{array}{l}\text { P- } \\
\text { value }\end{array}$ \\
\hline Age & 1.03 & $0.99-1.06$ & 0.180 & & & \\
\hline CA724 & 0.85 & $0.73-0.96$ & 0.010 & 0.83 & $0.71-$ & 0.004 \\
\hline Mitotic Count & 2.33 & $1.36-3.29$ & 0.080 & 4.24 & $3.02-$ & 0.020 \\
\hline Ki67 & 1.04 & $1.00-1.07$ & & & 5.45 & \\
\hline CD117 & 2477062603.06 & $2477023213.96-$ & 1.000 & & & \\
\hline Note: OR, odds ratio;95\% Cl,95\% confidence interval. & & & & & \\
\hline
\end{tabular}


Table 4

Prediction Efficiencies of Preoperative and Postoperative Clinical Models and Radiomics Nomogram

\begin{tabular}{|c|c|c|c|c|c|c|c|c|}
\hline & & AUC & $95 \% \mathrm{Cl}$ & Accuracy & Sensitivity & Specificity & PPV & NPV \\
\hline \multirow{2}{*}{$\begin{array}{l}\text { Preoperative } \\
\text { Clinical } \\
\text { Model } \\
\text { (CA724) }\end{array}$} & $\begin{array}{l}\text { Training } \\
\text { Set }\end{array}$ & 0.699 & $\begin{array}{l}0.586- \\
0.797\end{array}$ & 0.713 & 0.848 & 0.529 & 0.709 & 0.720 \\
\hline & $\begin{array}{l}\text { Validation } \\
\text { Set }\end{array}$ & 0.509 & $\begin{array}{l}0.307- \\
0.709\end{array}$ & 0.615 & 0.800 & 0.364 & 0.632 & 0.571 \\
\hline \multirow{2}{*}{$\begin{array}{l}\text { Postoperative } \\
\text { Clinical } \\
\text { Model } \\
\text { (CA724 and } \\
\text { Mitotic } \\
\text { Count) }\end{array}$} & $\begin{array}{l}\text { Training } \\
\text { Set }\end{array}$ & 0.770 & $\begin{array}{l}0.663- \\
0.857\end{array}$ & 0.763 & 0.870 & 0.618 & 0.755 & 0.778 \\
\hline & $\begin{array}{l}\text { Validation } \\
\text { Set }\end{array}$ & 0.606 & $\begin{array}{l}0.397- \\
0.790\end{array}$ & 0.615 & 0.600 & 0.636 & 0.692 & 0.538 \\
\hline \multirow{2}{*}{$\begin{array}{l}\text { Preoperative } \\
\text { Radiomics } \\
\text { Nomogram }\end{array}$} & $\begin{array}{l}\text { Training } \\
\text { Set }\end{array}$ & 0.913 & $\begin{array}{l}0.829- \\
0.964\end{array}$ & 0.863 & 0.891 & 0.824 & 0.872 & 0.848 \\
\hline & $\begin{array}{l}\text { Validation } \\
\text { Set }\end{array}$ & 0.715 & $\begin{array}{l}0.506- \\
0.873\end{array}$ & 0.769 & 0.800 & 0.727 & 0.800 & 0.727 \\
\hline \multirow{2}{*}{$\begin{array}{l}\text { Postoperative } \\
\text { Radiomics } \\
\text { Nomogram }\end{array}$} & $\begin{array}{l}\text { Training } \\
\text { Set }\end{array}$ & 0.919 & $\begin{array}{l}0.836- \\
0.968\end{array}$ & 0.863 & 0.935 & 0.765 & 0.843 & 0.897 \\
\hline & $\begin{array}{l}\text { Validation } \\
\text { Set }\end{array}$ & 0.679 & $\begin{array}{l}0.468- \\
0.847\end{array}$ & 0.769 & 0.800 & 0.727 & 0.800 & 0.727 \\
\hline
\end{tabular}

Because VOIs of tri-phase CE-CT were analyzed, we developed arterial phase (A-phase), venous phase (Vphase), delayed phase (D-phase), and tri-phase combined radiomics algorithms. This study calculated radiomics scores that could be used to predict GISTs with the KIT exon 11 mutation. Patients without the KIT exon 11 mutation generally had lower radiomics scores than patients with the KIT exon 11 mutation. The selected radiomics features of the A-phase, V-phase, D-phase, and combined radiomics algorithms are shown in Fig. 2. The prediction efficiencies of the radiomics algorithms to distinguish GISTs with the KIT exon 11 mutation in the training and validation sets are shown in Table 3. The AUCs of the radiomics algorithms with the training and validation sets were 0.773 and 0.709 , for the A-phase, 0.745 and 0.558 for the V-phase, 0.738 and 0.600 for the D-phase, and 0.826 and 0.836 for the combined radiomics algorithms respectively. These results show that the predictive performance of the combined radiomics algorithm was better than that of any of the single-phase algorithms. 
Table 3

Prediction Efficiencies of Radiomics Algorithm with the Training and Validation Sets

\begin{tabular}{|c|c|c|c|c|c|c|c|c|}
\hline & & AUC & $95 \% \mathrm{Cl}$ & Accuracy & Sensitivity & Specificity & PPV & NPV \\
\hline \multirow{2}{*}{$\begin{array}{l}\text { A-phase } \\
\text { Radiomics } \\
\text { Algorithm }\end{array}$} & $\begin{array}{l}\text { Training } \\
\text { Set }\end{array}$ & 0.773 & $\begin{array}{l}0.666- \\
0.859\end{array}$ & 0.725 & 0.783 & 0.647 & 0.750 & 0.688 \\
\hline & $\begin{array}{l}\text { Validation } \\
\text { Set }\end{array}$ & 0.709 & $\begin{array}{l}0.499- \\
0.869\end{array}$ & 0.807 & 0.933 & 0.636 & 0.778 & 0.875 \\
\hline \multirow{2}{*}{$\begin{array}{l}\text { V-phase } \\
\text { Radiomics } \\
\text { Algorithm }\end{array}$} & $\begin{array}{l}\text { Training } \\
\text { Set }\end{array}$ & 0.745 & $\begin{array}{l}0.635- \\
0.836\end{array}$ & 0.687 & 0.565 & 0.853 & 0.839 & 0.592 \\
\hline & $\begin{array}{l}\text { Validation } \\
\text { Set }\end{array}$ & 0.558 & $\begin{array}{l}0.351- \\
0.750\end{array}$ & 0.731 & 0.867 & 0.546 & 0.722 & 0.750 \\
\hline \multirow{2}{*}{$\begin{array}{l}\text { D-phase } \\
\text { Radiomics } \\
\text { Algorithm }\end{array}$} & $\begin{array}{l}\text { Training } \\
\text { Set }\end{array}$ & 0.738 & $\begin{array}{l}0.628- \\
0.830\end{array}$ & 0.688 & 0.674 & 0.706 & 0.756 & 0.615 \\
\hline & $\begin{array}{l}\text { Validation } \\
\text { Set }\end{array}$ & 0.600 & $\begin{array}{l}0.391- \\
0.785\end{array}$ & 0.615 & 0.533 & 0.727 & 0.727 & 0.533 \\
\hline \multirow{2}{*}{$\begin{array}{l}\text { Combined } \\
\text { Radiomics } \\
\text { Algorithm }\end{array}$} & $\begin{array}{l}\text { Training } \\
\text { Set }\end{array}$ & 0.826 & $\begin{array}{l}0.725- \\
0.902\end{array}$ & 0.763 & 0.696 & 0.853 & 0.865 & 0.674 \\
\hline & $\begin{array}{l}\text { Validation } \\
\text { Set }\end{array}$ & 0.836 & $\begin{array}{l}0.640- \\
0.951\end{array}$ & 0.808 & 0.800 & 0.818 & 0.857 & 0.750 \\
\hline
\end{tabular}

The preoperative and postoperative clinical models and combined radiomics algorithm were incorporated into the multivariate logistic regression analysis to generate preoperative and postoperative radiomics nomograms, respectively (Fig. 4A,D). The performances of preoperative and postoperative radiomics nomograms in distinguishing the patients with the KIT exon 11 mutation in the training and validation sets are shown in Table 4. For the preoperative radiomics nomogram with the training and validation sets, the AUCs were 0.913 and 0.715 respectively; for postoperative radiomics nomogram with the training and validation sets, the AUCs were 0.919 and 0.679 respectively. The ROC curves for the training and validation sets were used to compare the prediction efficiencies of the combined radiomics algorithm and preoperative and postoperative radiomics nomograms (Fig. 3A,B). Interestingly, with the training set, the preoperative and postoperative radiomics nomograms both had higher AUCs than the combined radiomics algorithm, whereas, with the validation set, the combined radiomics algorithm had a higher AUC than the three models.

The calibration curves of the combined radiomics algorithm(Fig. $5 C, D ; P=0.781$ and 0.534 )and preoperative(Fig. 4B,C; $P=0.838$ and 0.730 )and postoperative (Fig. $4 \mathrm{E}, \mathrm{F} ; \mathrm{P}=0.899$ and 0.495 ) radiomics nomograms showed good calibration with the nonsignificant Hosmer-Lemeshow test statistic with the training and validation sets respectively. The decision curve analysis (DCA) showed that the combined radiomics algorithm produced the highest overall net benefit compared with that of the preoperative or postoperative radiomics nomograms in predicting the KIT 11 mutation in the validation set across most of 
the range of reasonable threshold probabilities. However, the DCA found opposite results with the training set (Fig. 5A,B).

\section{Discussion}

The specific KIT 11 mutation is associated with the clinical response to targeted therapy of GISTs $(1,2,5)$. Selecting the targeted therapies based on the GIST genotypes has made precision medicine for GISTs possible. Radiogenomics approaches decode image phenotypes using radiomics to predict the genotypes of tumors, and they have been applied successfully in precision medicine studies $(11,17)$. In this retrospective two-center study, the aim was to develop a personalized radiomics method that could distinguish GISTs with the KIT exon 11 mutation by tri-phase CE-CT. We developed and validated clinical models, A-phase, V-phase, D-phase, and combined radiomics algorithms, and preoperative and postoperative radiomics nomograms. The results showed that the combined radiomics algorithm had a well-calibrated clinical benefit and the best ability to distinguish GISTs with the KIT exon 11 mutation in the validation set, which indicated that radiomics had accurate and reproducible value for predicting GISTs with the KIT exon 11 mutation genotype.

In a previous study, the clinical model was used to predict the biological behaviors of GISTs (25), and genotypes have been shown to be closely related to certain clinical characteristics (26-28). However, the relationship between some clinical characteristics and the KIT 11 mutation remains controversial $(26,27,29$, 30). Most of the previous studies showed that GISTs with the KIT exon 11 mutation usually had high percentages of mitotic count and/or poor prognosis $(26,27,30)$. The relationship between CA724 and GIST genotypes has not been reported until now. We found that, in the training set, the mitotic count and CA724 were correlated with the GISTs with the KIT exon 11 mutation. However, the preoperative and postoperative clinical models had relatively lower predictive effectiveness (AUCs $=0.509$ and 0.606 ) with the validation set than all the other models tested. This result indicated that the models based solely on clinical characteristics made only limited contributions to the discrimination performance. The roles of mitotic count and CA724 for differentiating the expression status of KIT exon 11 need further studies.

The extraction of high-dimensional features from medical images by radiomics has emerged as a promising approach for predicting the characteristic of tumors $(11,13,15,17)$. Radiomics is more comprehensive than morphological visual analysis and can quantitatively reveal tumor heterogeneity. Various types of medical images can be analyzed to help with diagnosis and treatment and support traditional diagnostic methods $(14,16,31,32)$. Considering that different mutation accompanied by different enhancement ratio, heterogeneous blood supply(33). Using VOIs extracted from tri-phase CE-CT images to construct a combined radiomics algorithm(11) can provide more representative information about a tumor when all available slices are considered (34), and may have better clinical application value and generalizability in discriminating mutation (34-36). In the present study, we extracted more advanced radiomics features and used relatively large data from two institutions compared with the CT texture analysis and single-center data in a previous study (11). We also used various evaluation tools, excellent radiomics feature selection methods, and machine-learning classifier to obtain the generalization and accuracy of the predictive model.

The radiomics algorithm was proven to be more effective than the clinical model. Next, we incorporated the clinical factors with the combined radiomics algorithm to build preoperative and postoperative radiomics 
nomograms. The AUC and DCA of the combined radiomics algorithm were compared with those of the preoperative and postoperative radiomics nomograms. The results showed that the combined radiomics algorithm had better predictive effectiveness and clinical applicability with the validation set than the radiomics nomograms, which indicated that the radiomics algorithm reflected molecular-level pathology better than the clinical factors and confirmed the enormous potential of the radiomics algorithm to distinguish GISTs with the KIT 11 mutation.

To prevent overfitting, the mRMR and LASSO regression methods were applied to the extracted features. For feature selection, we used mRMR, which is a new feature screening method (31), to screen radiomics features that had the fewest redundancies and most credible coefficients. LASSO is a feature screening method for selecting the predominant features of both ridge regression and subset screening (37). We used GLM as a machine-learning classifier because it was reported to produce the best screening performance among the 11 machine-learning methods that were tested (32). With application of the excellent feature selection methods and machine-learning classifier, the combined radiomics algorithm satisfactorily distinguished GISTs with and without the KIT exon 11 mutation.

The development of targeted therapy and genetic analysis have made precision medicine for GISTs feasible $(5,11,27,28)$. The most common imatinib-target mutation is the KIT exon 11 mutation, and patients with this mutation can benefit from standard imatinib therapy $(2,4,5,10)$. The relatively rare patients without the KIT exon 11 mutation require further genetic analysis to identify multiple effects of different treatments $(1,2,4-$ 6). The combined radiomics algorithm developed in this study will provide an alternative to biopsies, which can produce inconsistent results due to tumor heterogeneity, and surgery, which some special patients cannot tolerate $(2,8,10)$, to distinguish GISTs with the KIT exon 11 mutation non-invasively. For patients who were evaluated as the KIT exon 11 mutation with the combined radiomics algorithm, clinicians could recommend the standard imatinib therapy. For patients who were evaluated not to have the KIT exon 11 mutation, using the combined radiomics algorithm would not delay precision treatment or cause any unnecessary financial burden.

Our study has several limitations. First, all data were collected retrospectively and limited to Chinese patients, so selection bias was inevitable. Second, although the data were from two centers, the proportion of samples that had been performed genetic analysis was small, possibly because of cost considerations, which highlights the need for and significance of radiomics analysis. These findings need to be validated in international, larger multi-institutional studies. Third, only CE-CT images (slice thickness of $5.0 \mathrm{~mm}$ ) were analyzed in this study. Whether radiomics could achieve a better performance on different slice thickness of $\mathrm{CE}-\mathrm{CT}$ is worth further investigation. Fourth, the hand-crafted feature requires time-consuming tumor boundary segmentation, deep learning radiomics could accurately and automatically detect and segment and achieve excellent performances (17). Lastly, this study focused only on GISTs with the KIT exon 11 mutation. GISTs without the KIT exon 11 mutation or secondary mutation could be explored in future work (4, 38).

\section{Conclusion}


We developed and validated several predictive models that could non-invasively distinguish GISTs with KIT exon 11 mutation based on tri-phase CE-CT images. The combined radiomics algorithm performed better than the other predictive models, which confirmed it as a potential predictor to supplement conventional approaches for selective genetic analysis and to support the clinical decision about imatinib therapy in the precision medicine of GISTs.

\section{List Of Abbreviations}

GISTs: gastrointestinal stromal tumors; A-phase: arterial phase; V-phase: venous phase; D-phase: delayed phase; CE-CT: contrast-enhanced computed tomography; LASSO: least absolute shrinkage and selection operator; mRMR: minimum redundancy maximum relevance; GLM: generalized linear model; AUC: area under the curve; ROC: receiver operating characteristic curve; Cl: confidence interval; PDGFRA: platelet-derived growth factor receptor alpha; TKIs: tyrosine kinase inhibitors; NCCN: National Comprehensive Cancer Network; VOI: volume of interest; ICCs: inter-/ intra-class correlation coefficients; GLCM: grey-level cooccurrence matrix; GLRLM: gray-level run length matrix; NIH: National Institutes of Health; DCA: decision curve analysis; CEA: carcinoembryonic antigen; CA199: carbohydrate antigen 199; CA125: carbohydrate antigen 125; CA724:carbohydrate antigen 724;OR:odds ratio; PPV: positive predictive value; NPV: negative predictive value.

\section{Declarations}

\section{Ethics approval and consent to participate}

Our Institutional Review Board (Medical Ethics Committee of the Affiliated Hospital of Qingdao University and the Shandong Provincial Hospital affiliated to Shandong First Medical University) approved this study and waived the need for informed consent from patients.

\section{Consent for publication}

All authors have read and approved the content and agree to submit for consideration for publication in the journal.

\section{Availability of data and materials}

All data generated or analysed during this study are included in this published article.

\section{Competing interests}

The authors declare that they have no competing interests.

\section{Funding}

This work was supported by National Natural Science Foundation of China (81770631).

\section{Author Contributions}


All authors have had access to the data and all drafts of the manuscript. Specifc contributions are as follows: design and supervision: $\mathrm{JZ}, \mathrm{XJ}$; development of methodology: $\mathrm{JZ}, \mathrm{XJ}, \mathrm{BL}, \mathrm{HW}$; collection and extraction of data: $\mathrm{BL}, \mathrm{SY}, \mathrm{YS}, \mathrm{HL}, \mathrm{ZZ}, \mathrm{JZ}, \mathrm{FH}$; analysis and interpretation of data (e.g., statistical analysis, algorithm and software development):SL; manuscript writing: LB, HW; final approval of manuscript: all authors.

\section{Acknowledgements}

We appreciate the help from other teammates.

\section{Author details}

${ }^{1}$ Department of General Surgery; The Affiliated Hospital of Qingdao University, Qingdao, Shandong, China; ${ }^{2}$ Department of Radiology; The Affiliated Hospital of Qingdao University, Qingdao, Shandong, China; ${ }^{3}$ Department of Radiology; Shandong Provincial Hospital Affiliated to Shandong First Medical University, Jinan, Shandong, China; ${ }^{4}$ Department of Pathology; The Affiliated Hospital of Qingdao University, Qingdao, Shandong, China

\section{References}

1. $M C H, C L C, G D D, C D B, M v M, H J$, et al. Kinase mutations and imatinib response in patients with metastatic gastrointestinal stromal tumor. 2003;21 \% J Journal of clinical oncology : official journal of the American Society of Clinical Oncology( $23 \% \mathrm{~J} J$ Clin Oncol).

2. $M v M, R L R, R S B, S B, M M B, K N$ G, et al. Soft Tissue Sarcoma, Version 1.2018, NCCN Clinical Practice Guidelines in Oncology. 2018;16 \% J Journal of the National Comprehensive Cancer Network : JNCCN(5 \%J J Natl Compr Canc Netw).

3. Edmonson JH, Marks RS, Buckner JC, Mahoney MR. Contrast of response to dacarbazine, mitomycin, doxorubicin, and cisplatin (DMAP) plus GM-CSF between patients with advanced malignant gastrointestinal stromal tumors and patients with other advanced I eiomyosarcomas. Cancer Invest. 2002;20(5-6):605-12.

4. Oppelt PJ, Hirbe AC, Van Tine BA. Gastrointestinal stromal tumors (GISTs): point mutations matter in management, a review. Journal of gastrointestinal oncology. 2017;8(3):466-73.

5. $\mathrm{MC} \mathrm{H}, \mathrm{K} \mathrm{O}, \mathrm{CL} C, \mathrm{D} \mathrm{H}, \mathrm{EC} \mathrm{B}, \mathrm{CD} F$, et al. Correlation of kinase genotype and clinical outcome in the North American Intergroup Phase III Trial of imatinib mesylate for treatment of advanced gastrointestinal stromal tumor: CALGB 150105 Study by Cancer and Leukemia Group B and Southwest Oncology Group. $2008 ; 26 \% J$ Journal of clinical oncology : official journal of the American Society of Clinical Oncology(33 $\% \mathrm{~J} J$ Clin Oncol).

6. P C, L Z, W Z, L S. Efficacy evaluation of imatinib treatment in patients with gastrointestinal stromal tumors: a meta-analysis. 2010;16 \% J World journal of gastroenterology(33 \% J World J Gastroenterol).

7. CL C, KV B, CR A, V K, RG M, PW P, et al. Pathologic and molecular features correlate with long-term outcome after adjuvant therapy of resected primary GI stromal tumor: the ACOSOG Z9001 trial. 2014;32(15):1563-70. 
8. GH K, JY A, CS G, M K, HK N, JH L, et al. Efficacy of Endoscopic Ultrasound-Guided Fine-Needle Biopsy in Gastric Subepithelial Tumors Located in the Cardia. 2019.

9. Chen T, Xu L, Dong X, Li Y, Yu J, Xiong W, et al. The roles of CT and EUS in the preoperative evaluation of gastric gastrointestinal stromal tumors larger than 2 cm. European Radiology. 2019;29(5):2481-9.

10. Li J, Ye Y, Wang J, Zhang B, Qin S, Shi Y, et al. Chinese consensus guidelines for diagnosis and management of gastrointestinal stromal tumor. Chinese journal of cancer research = Chung-kuo yen cheng yen chiu. 2017;29(4):281-93.

11. Xu F, Ma X, Wang Y, Tian Y, Tang W, Wang M, et al. CT texture analysis can be a potential tool to differentiate gastrointestinal stromal tumors without KIT exon 11 mutation. Eur J Radiol. 2018;107:90-7.

12. Choi H. Response evaluation of gastrointestinal stromal tumors. The oncologist. 2008;13 Suppl 2:4-7.

13. Lambin P, Leijenaar RTH, Deist TM, Peerlings J, de Jong EEC, van Timmeren J, et al. Radiomics: the bridge between medical imaging and personalized medicine. Nature reviews Clinical oncology. 2017;14(12):749-62.

14. Nie P, Yang G, Wang Z, Yan L, Miao W, Hao D, et al. A CT-based radiomics nomogram for differentiation of renal angiomyolipoma without visible fat from ho mogeneous clear cell renal cell carcinoma. Eur Radiol. 2020;30(2):1274-84.

15. Liu S, He J, Liu S, Ji C, Guan W, Chen L, et al. Radiomics analysis using contrast-enhanced CT for preoperative prediction of occult peritoneal metast asis in advanced gastric cancer. Eur Radiol. 2020;30(1):239-46.

16. Nie P, Yang G, Wang N, Yan L, Miao W, Duan Y, et al. Additional value of metabolic parameters to PET/CTbased radiomics nomogram in predicting lymphovascu lar invasion and outcome in lung adenocarcinoma. Eur J Nucl Med Mol Imaging. 2020.

17. Zhao W, Yang J, Ni B, Bi D, Sun Y, Xu M, et al. Toward automatic prediction of EGFR mutation status in pulmonary adenocarcinoma with 3D deep learning. Cancer medicine. 2019;8(7):3532-43.

18. G C, M S, imaging MFJMr. Influence of MRI acquisition protocols and image intensity normalization methods on texture classification. 2004;22(1):81-91.

19. P G, medicine TLJMri. Textural analysis of contrast-enhanced MR images of the breast. 2003;50(1):92-8.

20. A D, A F-R, D VDV, analysis MHJMi. Three-dimensional solid texture analysis in biomedical imaging: review and opportunities. 2014;18(1):176-96.

21. F O, F F, C N, N A, Radiology BIJ. Validation of A Method to Compensate Multicenter Effects Affecting CT Radiomics. 2019;291(1):53-9.

22. JP F, N C, YI S, WD T, I A, PA C, et al. Harmonization of cortical thickness measurements across scanners and sites. 2018;167:104-20.

23. A I, D S, GV R, Oncology PKJJocoojotASoC. How to build and interpret a nomogram for cancer prognosis. 2008;26(8):1364-70.

24. AJ V, Making EEJMdmaijotSfMD. Decision curve analysis: a novel method for evaluating prediction models. 2006;26(6):565-74.

25. Gold JS, G?nen M, Guti”|'Irrez A, Broto JM, Garc“"aa-del-Muro X, Smyrk TC, et al. Development and validation of a prognostic nomogram for recurrence-free survival after complete surgi cal resection of 
localised primary gastrointestinal stromal tumour: a retrospective analysis. The Lancet Oncology. 2009;10(11):1045-52.

26. Baskin Y, Kocal GC, Kucukzeybek BB, Akbarpour M, Kayacik N, Sagol O, et al. PDGFRA and KIT Mutation Status and Its Association With Clinicopathological Properties, Including DOG1. Oncol Res. 2016;24(1):41-53.

27. Quek R, Farid M, Kanjanapan Y, Lim C, Tan IB, Kesavan S, et al. Prognostic significance of KIT exon 11 deletion mutation in intermediate-risk gastrointestinal stromal tumor. Asia Pac J Clin Oncol. 2017;13(3):115-24.

28. Wozniak A, Rutkowski P, Piskorz A, Ciwoniuk M, Osuch C, Bylina E, et al. Prognostic value of KIT/PDGFRA mutations in gastrointestinal stromal tumours (GIST): Polish Clinical GIST Registry experience. Annals of oncology : official journal of the European Society for Medical Oncology. 2012;23(2):353-60.

29. Vitiello GA, Bowler TG, Liu M, Medina BD, Zhang JQ, Param NJ, et al. Differential immune profiles distinguish the mutational subtypes of gastrointestinal stromal tumor. The Journal of clinical investigation. 2019;129(5):1863-77.

30. Capelli L, Petracci E, Quagliuolo V, Saragoni L, Colombo P, Morgagni P, et al. Gastric GISTs: Analysis of cKit, PDGFRA and BRAF mutations in relation to prognosis and clinical pathological characteristics of patients - A GIRCG study. Eur J Surg Oncol. 2016;42(8):1206-14.

31. W L, L Z, D D, M F, Q D, S L, et al. Radiomic analysis for preoperative prediction of cervical lymph node metastasis in patients with papillary thyroid carcinoma. 2019;118:231-8.

32. Parmar C, Grossmann P, Rietveld D, Rietbergen MM, Lambin P, Aerts HJ. Radiomic Machine-Learning Classifiers for Prognostic Biomarkers of Head and Neck Cancer. Front Oncol. 2015;5:272.

33. Yin YQ, Liu CJ, Zhang B, Wen Y, Yin Y. Association between CT imaging features and KIT mutations in small intestinal gastrointestinal stromal tumors. Scientific reports. 2019;9(1):7257.

34. F N, R K, B G, radiology GVJEjo. Assessment of tumor heterogeneity by CT texture analysis: can the largest cross-sectional area be used as an alternative to whole tumor analysis? 2013;82(2):342-8.

35. Zhou C, Duan X, Zhang X, Hu H, Wang D, Shen J. Predictive features of CT for risk stratifications in patients with primary gastrointestinal stromal tumour. Eur Radiol. 2016;26(9):3086-93.

36. Jiang Z, Zhang J, Li Z, Liu Y, Wang D, Han G. A meta-analysis of prognostic value of KIT mutation status in gastrointestinal stromal tumors. OncoTargets and therapy. 2016;9:3387-98.

37. Gui J, Li H. Penalized Cox regression analysis in the high-dimensional and low-sample size settings, with applicat ions to microarray gene expression data. Bioinformatics. 2005;21(13):3001-8.

38. CR A, P B, T G, K A, G H, B K, et al. Acquired resistance to imatinib in gastrointestinal stromal tumor occurs through secondary gene mutation. $2005 ; 11 \% \mathrm{~J}$ Clinical cancer research : an official journal of the American Association for Cancer Research(11 \% J Clin Cancer Res).

\section{Figures}




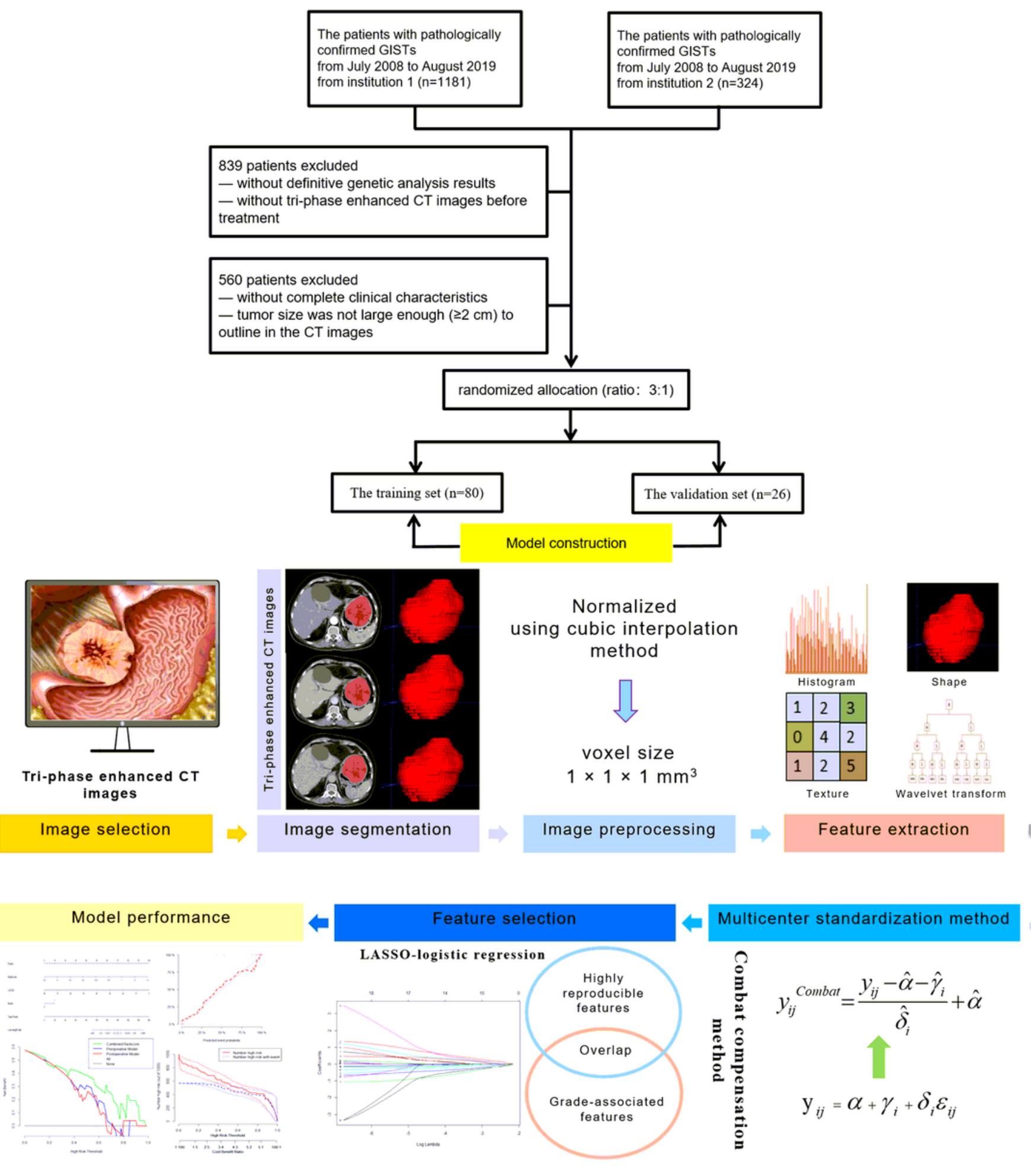

Figure 1

Workflow process for radiomics processing and analysis in this study. 
a

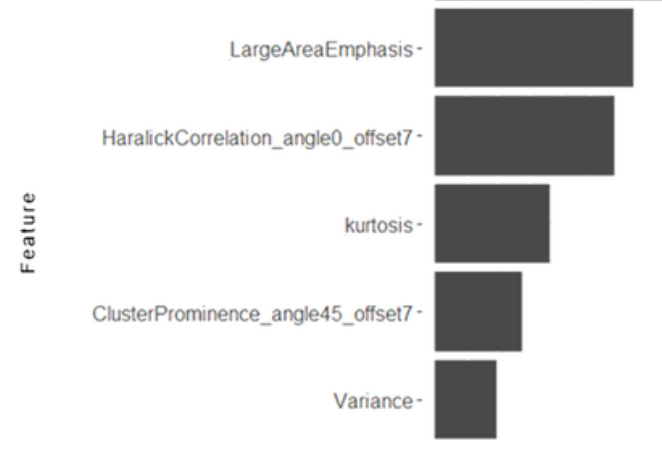

LowintensityLargeAreaEmphasis

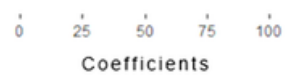

C

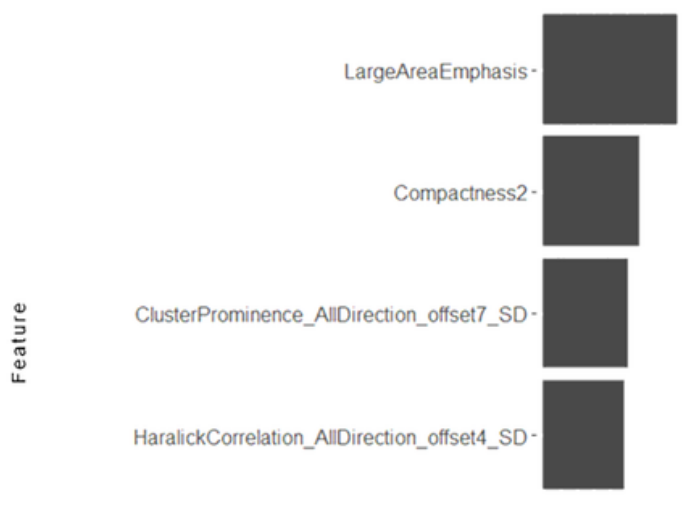

HighGreyLevelRunEmphasis_AllDirection_offset1_SD

¿. 25 so 50 is 100 Coefficients b

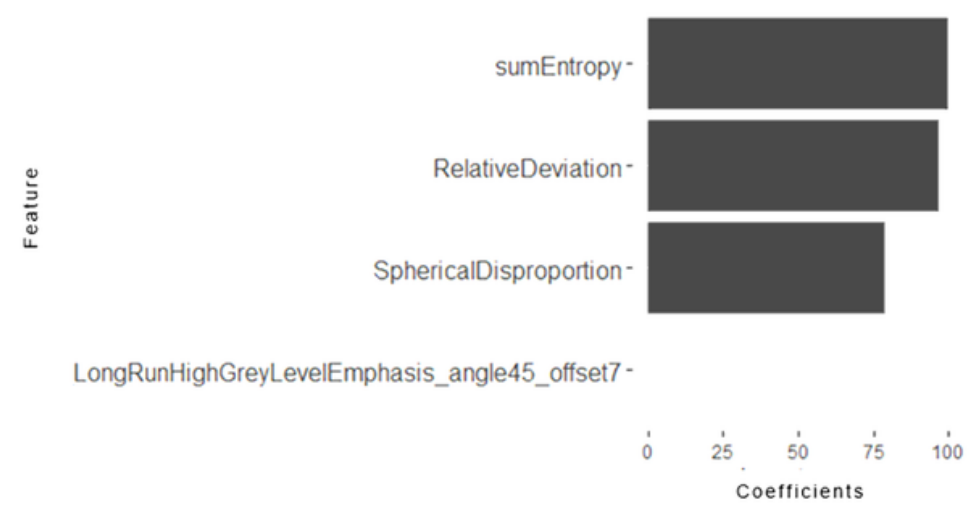

d

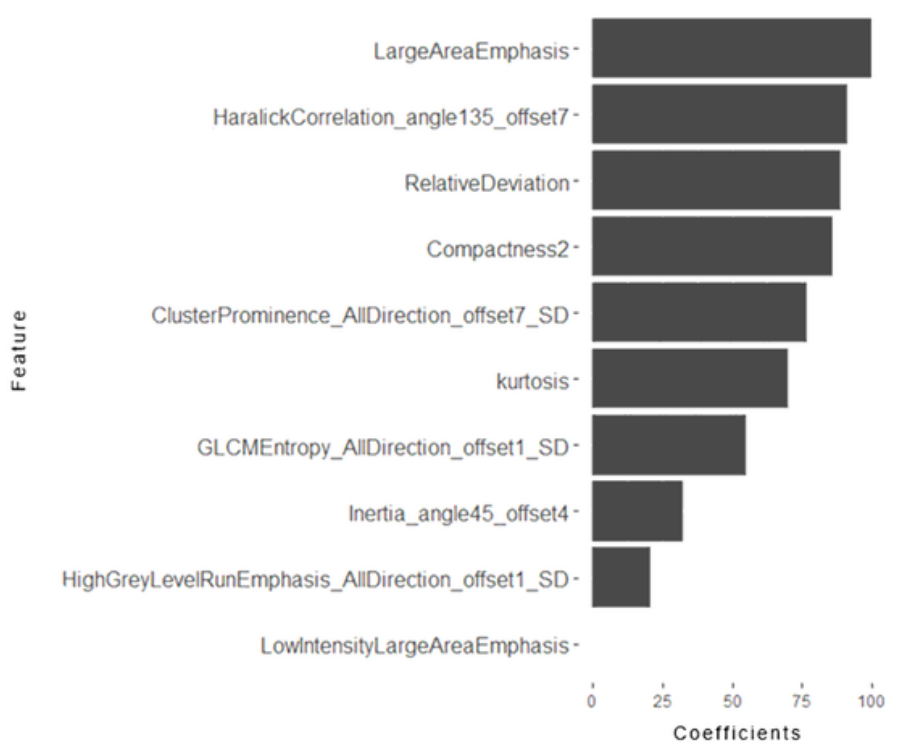

\section{Figure 2}

The selected radiomics features and their coefficients of the A-phase (a), V-phase (b), D-phase, (c) and combined (d) radiomics algorithms. X-axis, coefficients of features with the LASSO logistic regression analysis; $y$-axis, features selected using the radiomics algorithms. 
a

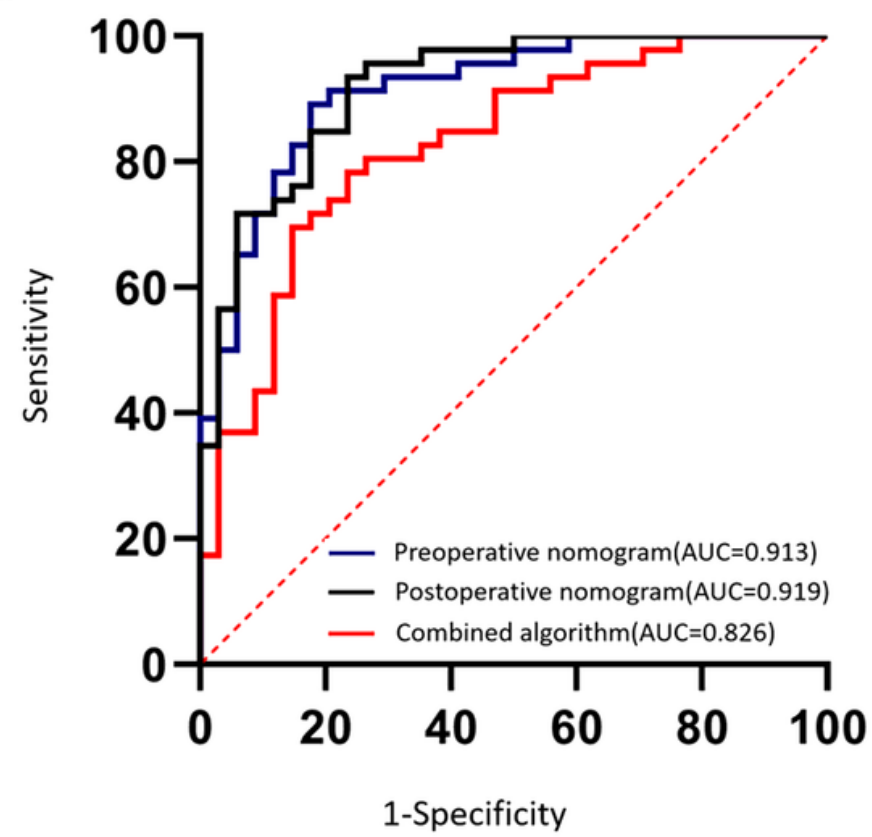

b

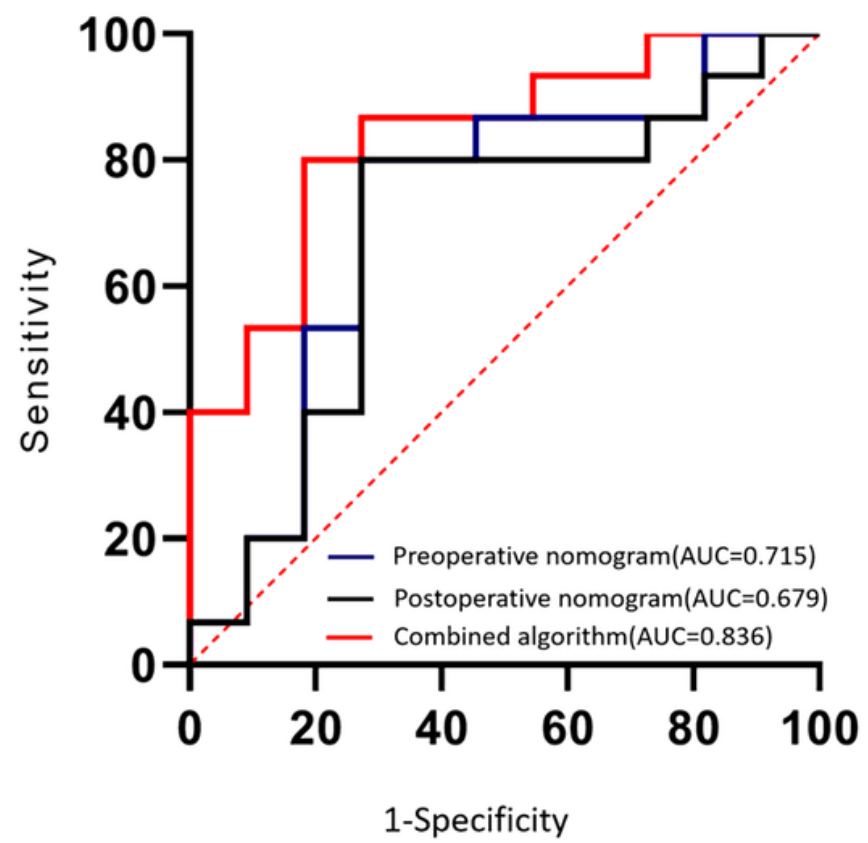

Figure 3

ROC curves for the combined radiomics algorithm, preoperative and postoperative radiomics nomograms with the training (a) and validation (b) sets.

a

Points

Rad-score

CA724

Total points

Possibility of KIT exon 11mutation

d

Points

Rad-score

CA724

Mitotic count

Total points

Possibility of KIT exon 11mutation b

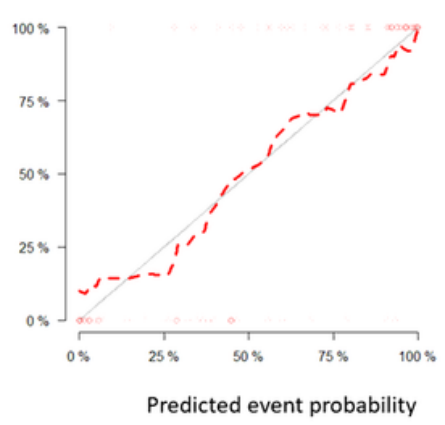

e

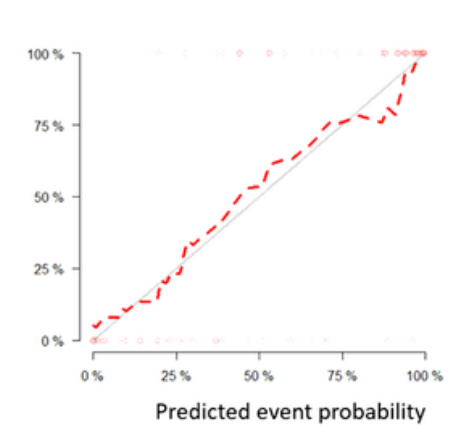

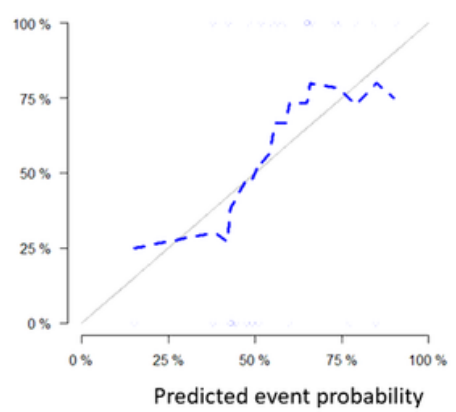

f

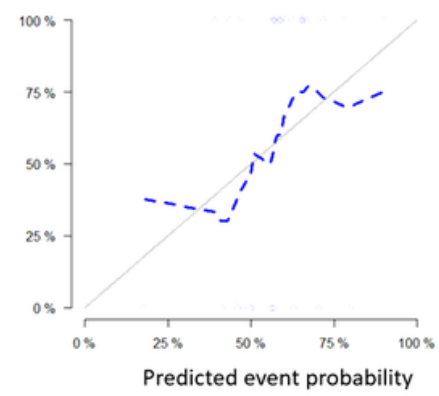

Figure 4

(a) Preoperative radiomics nomogram for predicting the KIT exon 11 mutation. Calibration curves of the preoperative radiomics nomogram with the training (b) and validation (c) sets. (d) Postoperative radiomics nomogram for predicting the KIT exon 11 mutation. Calibration curves of postoperative radiomics nomogram with the training (e) and validation ( $F$ ) sets. 
a

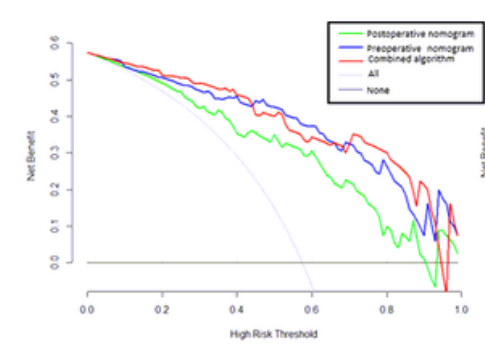

b

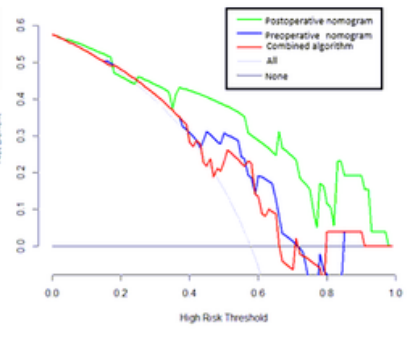

c

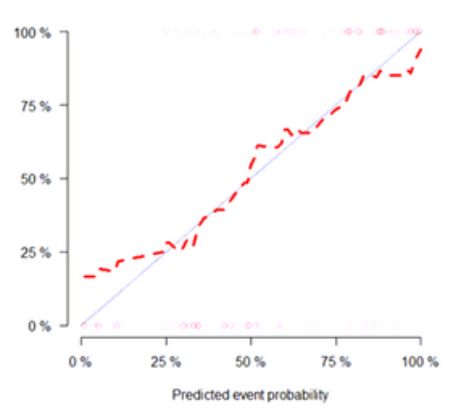

d

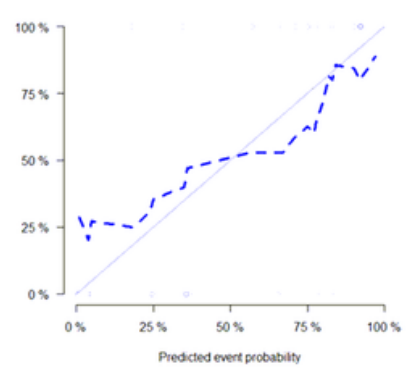

e

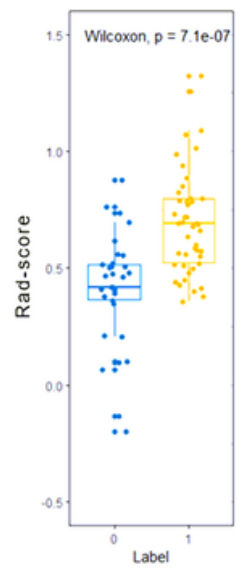

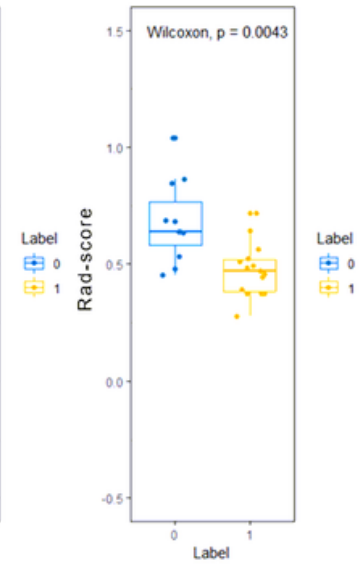

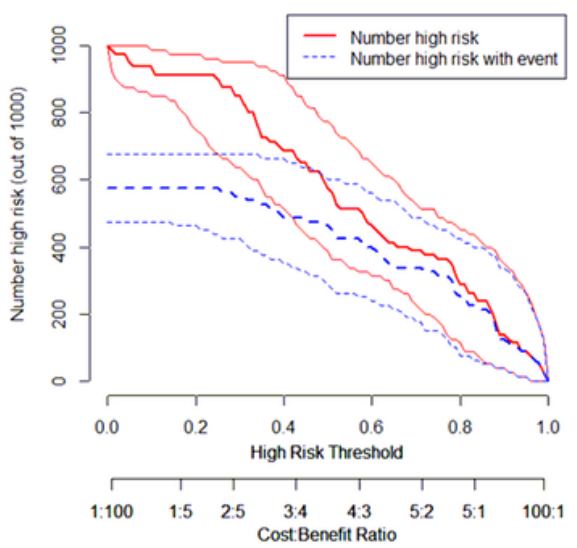

\section{Figure 5}

Decision curve analysis (DCA) for the combined radiomics algorithm, and preoperative and postoperative radiomics nomograms with the training (a) and validation (b) sets. The combined radiomics algorithm had the highest net benefit when the threshold probability was between 0.3 and 1 in the validation set, compared with the simple strategies of no patients (horizontal black line) or all patients (grey line) and other methods. Calibration curves of combined radiomics algorithm with the training (c) and validation (d) sets. (e) Validation of radiomics scores (rad-scores). Blue and yellow show the actual classification. Low degree of mix of blue and yellow indicates good predictive ability of the rad-score; complete separation of blue and yellow indicates that the rad-score can be well classified. (f) Clinical impact curves of the combined radiomics algorithm. Number high risk with event (blue curve) is the number of true positives at each threshold probability; number high risk (red curve) indicates the number of patients classified as positive by the algorithm at each threshold probability. 